Les marchands espagnols dans l'élite dirigeante du Conseil des bourgeois de Nantes au milieu du XVIe siècle

\title{
Guy Saupin
}

\section{(apenEdition}

\section{Journals}

\section{Édition électronique}

URL : http://journals.openedition.org/abpo/1054

DOI : $10.4000 /$ abpo.1054

ISBN : 978-2-7535-1500-0

ISSN : 2108-6443

Éditeur

Presses universitaires de Rennes

Édition imprimée

Date de publication : 20 décembre 2005

Pagination : 157-181

ISBN : 978-2-7535-0243-7

ISSN : 0399-0826

\section{Référence électronique}

Guy Saupin, «Les marchands espagnols dans l'élite dirigeante du Conseil des bourgeois de Nantes au milieu du XVIe siècle ", Annales de Bretagne et des Pays de l'Ouest [En ligne], 112-4 | 2005, mis en ligne le 20 décembre 2007, consulté le 19 avril 2019. URL : http://journals.openedition.org/abpo/1054 DOI : 10.4000/abpo.1054 


\title{
Les marchands espagnols dans l'élite dirigeante du Conseil des bourgeois de Nantes au milieu du XVI ${ }^{\mathrm{e}}$ siècle
}

\author{
Guy SAUPIN \\ Professeur d'histoire moderne \\ CRHIA - Université de Nantes
}

Depuis le milieu du XIV ${ }^{\mathrm{e}}$ siècle, les bourgeois de Nantes ont été associés par les ducs de Bretagne à la gestion financière de la défense de la ville. En 1410 , mais surtout en 1420, Jean V renforça cette participation en l'intégrant dans un conseil de bourgeois au côté des gens d'Église et de justice, l'ensemble étant placé sous l'autorité du capitaine, représentant suprême de l'autorité ducale ${ }^{1}$. Au cours d'une longue évolution, difficile à dater faute d'archives, ce conseil a pris l'habitude de fonctionner en assemblée générale intéressant prioritairement l'élite urbaine. C'est ce qui ressort du dernier et seul registre de délibération conservé ${ }^{2}$, couvrant la période août 1555-février 1563. Les relations entre les grands marchands et le capitaine s'étant détériorées suite à l'autoritarisme nouveau affiché par le comte René de Sanzay, ces derniers parvinrent à obtenir du roi François II en janvier 1559 (1560) la création d'un corps de ville, avec un maire, dix échevins et un procureur du roi syndic, doté d'un pouvoir de police urbaine beaucoup plus fort ${ }^{3}$. Cette remise en cause de l'équilibre institutionnel ancien dressa contre la nouvelle municipalité tous les autres corps dotés d'autorité, ce qui explique que le nouvel échevinage ne put être véritablement établi qu'en décembre 1564. Dans ce groupe de pression, des membres du groupe hispano-nantais, formé principalement d'immigrants venus de Vieille-Castille ${ }^{4}$, ont joué un rôle majeur.

1. LE MENe, Michel, «Temps de prospérité et temps des illusions », dans Bors, Paul (dir.), Histoire de Nantes, Toulouse, Privat, 1977, p. 82-88; id., La ville de Nantes au Xve siècle, DES, Rennes, 1959; VAllHEn, Jacques, Le Conseil des bourgeois de Nantes, Thèse de droit, dact., Rennes, 1961, 3 tomes.

2. Arch. mun. de Nantes, BB 4. Jacques Vailhen en a assuré la transcription dans le tome 3 de sa thèse.

3. VAILHEN, Jacques, L'Établissement de la première mairie à Nantes, DES, Rennes, 1960.

4. RAIMBAUD, Élodie, Les étrangers en Bretagne d'après les lettres de naturalité, XVIe-1661, 
Si l'absence de règlement de l'assemblée générale des " nobles bourgeois, manans et habitans " postule par voie de conséquence la reconnaissance du droit d'entrée à n'importe quel chef de feu de la ville de Nantes et de ses faubourgs, les conditions sociales et culturelles définissant la vie politique urbaine $\mathrm{au} \mathrm{XVI}^{\mathrm{e}}$ siècle rendaient cette hypothèse tout à fait improbable, tout en garantissant une bonne intégration de l'élite artisanale à la vie municipale pour ceux qui le désiraient. Une approche quantitative de la participation s'avère quasiment impossible puisque les greffiers n'inscrivaient que les noms des participants les plus notables, ceux qui servaient à fonder la qualité de l'assemblée délibérante en conformité avec les références de la culture oligarchique de la représentation qui prévalait à tous les niveaux du politique, depuis les états généraux du royaume jusqu'aux assemblées villageoises en incluant naturellement les municipalités urbaines. Ainsi, le registre du Conseil des bourgeois de Nantes se contente de fournir des listes variant entre une vingtaine et une cinquantaine de noms sans que l'on sache exactement quelle proportion des présents est pointée. Il n'est pas sûr que le dépassement fut d'ailleurs très grand car la fréquence des réunions exigeait beaucoup de temps libre des intéressés, donc une situation économique bien établie avec la faculté de déléguer ses affaires à un commis ou un employé digne de confiance, ce qui n'était pas à la portée du plus grand nombre.

Toutefois, à la simple lecture de tous ces procès-verbaux, il est évident que tous ces membres actifs du Conseil des bourgeois n'avaient pas la même envergure, ni le même poids politique dans cette assemblée délibérante. Certains noms reviennent plus que d'autres, des quasi-permanents côtoient des participants irréguliers ${ }^{5}$. Cette inégalité de prestation, relevant à la fois du degré d'investissement personnel et de la sélection interne à l'œuvre dans cette oligarchie dirigeante, nous renvoie vers la distinction classique entre un noyau dur, centre de détermination de la politique municipale, et une première couronne forte de son rôle essentiel de cautionnement des décisions stratégiques ${ }^{6}$. Plusieurs familles d'origine castillane, dont l'arrivée à Nantes est plus ou moins ancienne, y ont joué un rôle essentiel.

Maîtrise, LE PAge, Dominique (dir.), université de Nantes, 2000. MATHOREz, Jules, " Notes sur les Espagnols à Nantes ", Bulletin hispanique, Bordeaux, 1912, t. 14, p. 3-4.

5. SAuPIN, Guy, "Les marchands dans le Conseil des bourgeois de Nantes au milieu du $\mathrm{XvI}^{\mathrm{e}}$ siècle ", dans Mélanges Jean Tanguy, Bretagnes, art, négoce et société de l'Antiquité à nos jours, Brest, Asso. Amis J. Tanguy, 1996, p. 229-239. Première approche du thème abordé dans cet article.

6. SAUPIN, Guy, "Les oligarchies municipales en France sous l'Ancien Régime : réflexion méthodologique sur l'analyse historique de leur reproduction à partir de l'exemple de Nantes ", dans PETITFRERE, Claude (dir.), Construction, reproduction et représentation des patriciats urbains de l'Antiquité au XX' siècle, Tours, CEHVI, 1999, p. 95-112; MouYSSET, Sylvie, Le Pouvoir dans la bonne ville. Les consuls de Rodez sous l'Ancien Régime, Rodez/Toulouse, Société des Lettres, Sciences et Arts de l'Aveyron/CNRS/Université Toulouse-Le Mirail, 2000; Bonin, Pierre, Bourgeois, bourgeoisie et habitanage dans les villes du Languedoc sous l'Ancien Régime, Aix-en-Provence, PU Aix-Marseille, 2005, chapitre IV : " Accéder au pouvoir ». 


\section{À la recherche du noyau dur à travers la hiérarchie des missions déléguées}

Afin de cerner la composition de ce cœur du pouvoir municipal, il nous a paru pertinent de partir de l'examen de la distribution des principales missions au sein du Conseil des bourgeois, laissant ainsi au cumul la faculté de faire émerger les personnalités les plus reconnues par les acteurs euxmêmes.

\section{Les députations vers l'extérieur}

Il nous semble essentiel de commencer par le recensement des députations envoyées par le Conseil en direction de toutes les autorités politiques avec lesquelles une ville comme Nantes devait compter pour son développement fonctionnel et son inscription dans le schéma corporatif privilégié du royaume. Être choisi comme représentant officiel de la ville vis-à-vis du monde extérieur, avec mandat de négociation de ses intérêts supérieurs, ne pouvait revenir qu'à des notables au prestige bien établi, parfaitement reconnus comme tels dans l'élite urbaine. Naturellement, l'existence de trois officiers municipaux - procureur des bourgeois, miseur (trésorier), contrôleur - chargés de traduire concrètement les décisions collectives, pesait sur cette distribution dans la mesure où leur fonction les prédisposait à assurer ces missions de représentation. Cependant, leur élection antérieure par l'ensemble des membres du Conseil, dans des réunions totalisant le plus grand nombre de participants, leur avait déjà permis d'obtenir cette reconnaissance de leurs pairs.

Les députations à la cour sont les plus significatives car il s'agit d'obtenir les faveurs royales sur les questions politiques les plus graves. Ainsi, le 25 octobre 1555, Jehan Le Lou l'aîné et le miseur Geoffroy Drouet sont missionnés " pour avoir prolongation des privilèges pour les francs fiefs, pour le collège, pour le parlement, aussi pour faire les guets, pour savoir l'opinion du roi, aussi qu'il est dû de l'argent au receveur des États pour l'abolition de l'imposition ${ }^{7}$ ". Le dossier est d'importance. En mars 1557, Le Lou est obligé de produire une lettre du roi car il n'est toujours pas remboursé de ses frais ${ }^{8}$.

Depuis l'édit du roi Henri II de mars 1554 transformant l'ancienne cour souveraine de justice provinciale en véritable parlement, la lutte fait rage entre Nantes et Rennes pour rompre avec le partage traditionnel des saisons d'hiver et d'été et pour obtenir la fixation totale de l'institution dans une seule ville. Les communautés députant aux États de Bretagne, consultées au printemps 1555, se sont partagées, sans nette majorité, reflétant les aires d'influence administratives ou commerciales des deux villes en

7. Arch. mun. de Nantes, BB $4, \mathrm{f}^{\circ} 6 \mathrm{r}^{\circ}$.

8. Arch. mun. de Nantes, BB $4, \mathrm{f}^{\circ} 92 \mathrm{v}^{\circ}$. 
compétition ${ }^{9}$. L'action des groupes de pression auprès du conseil du roi, incluant de solides offres financières, est donc devenue essentielle. Nantes a gagné la première manche (édit de juin 1557) avant de perdre la seconde (édit de mars 1561). Les questions d'exemption fiscale comme l'extension du privilège d'exonération des francs-fiefs aux notables des faubourgs ou le rachat de la traite foraine ${ }^{10}$, la création d'un collège pour renforcer le pôle culturel urbain déjà honoré de la présence d'une université, et la régulation du service milicien soumis à la pression du nouveau capitaine ${ }^{11}$, sont autant de questions sensibles d'importance majeure. Le 21 juin 1557, afin de défendre la fixation complète du parlement de Bretagne à Nantes, le miseur et Michel Loriot sont envoyés en cour malgré la faiblesse de la miserie obligée de recourir à l'emprunt ${ }^{12}$. Le 31 décembre 1557, Michel Loriot est renvoyé à la cour, toujours pour garantir l'attribution exclusive de la cour souveraine ${ }^{13}$.

La mort accidentelle d'Henri II ayant relancé la compétition, le dossier du parlement est suivi avec une extrême attention car les surenchères financières s'avèrent stratégiques ${ }^{14}$. Pour les questions primordiales, l'usage est d'élire plus de délégués qu'il n'en faut afin de faire face aux éventuelles indisponibilités. Le 30 août 1560, les bourgeois " constituent à leurs procureurs pour comparaître devant le roi et messieurs de son privé conseil pour faire les requêtes telles qu'ils verront par conseil; Me Julien Dauffy (procureur des bourgeois), Bertrand Duchesne, Michel Loryot, et deux d'eux avec pouvoir de substituer et faire telles offres qu'il sera requis pour l'utilité de la ville audit dessus de ceux de la ville de Rennes ainsi que l'on a fait par ci-devant ». Lorsque les Rennais ont obtenu, grâce au soutien duc d'Étampes, gouverneur de Bretagne, le transfert du parlement de Nantes à Rennes par lettres patentes de Charles IX du 4 mars 1561, le conseil organise la contre-attaque. L'assemblée du premier mai 1561 renvoie les mêmes munis de 200 écus avancés par Yvon Rocaz et Antoine de Mirande, deux puissants marchands d'origine espagnole ${ }^{15}$.

Nantes était une des villes qui bénéficiaient du privilège de députation aux États de Bretagne pour la formation de l'ordre du tiers état. Vu son poids

9. SAUPIN, Guy, «La perception du réseau urbain dans le conflit entre Nantes et Rennes pour la localisation du parlement au milieu du XVI ${ }^{\mathrm{e}}$ siècle (1541-1565) ", dans GuIGNET, Philippe, HiernaRd, Jean (éd.), Les réseaux urbains dans le Centre-Ouest atlantique de l'Antiquité à nos jours, Poitiers, Mémoires de la Société des Antiquaires de l'Ouest, 1995, p. $145-172$.

10. VAILHEN, Jacques, op. cit., p. 165-170.

11. Ibidem, p. 406-414.

12. Arch. mun. de Nantes, BB $4, \mathrm{f}^{\circ} 60$.

13. Arch. mun. de Nantes, BB $4, f^{\circ} 84 r^{\circ}$. «Et sera fait mémoires et injonctions que portera ledit Loryot à la cour pour se gouverner et faire selon, ces mémoires sont pour obtenir du roi, si être peut, homologation de la taxe faite par les habitants sur les marchandises pour le recouvrement de la somme de 28000 livres pour le Parlement et autres leurs affaires".

14. Arch. mun. de Nantes, BB $4, \mathrm{f}^{\circ} 242 \mathrm{v}^{\circ}$.

15. Arch. mun. de Nantes, BB $4, \mathrm{f}^{\circ} 282 \mathrm{v}^{\circ}$. 
institutionnel mais surtout économique, sa voix comptait dans cette assemblée provinciale, dans une perpétuelle émulation avec Rennes, sa rivale de toujours. Le Conseil des bourgeois y déléguait deux ou trois de ses membres. Pour la session de l'automne 1557, il s'agit du procureur des bourgeois, Mathurin Vivien et du miseur Geoffroy Drouet ${ }^{16}$; pour celle de septembre 1558 tenue à Dinan, sont commis, outre le procureur des bourgeois Jean Duluc, François Salmon et Jean Le Lou l'aîné. Ce dernier fut également choisi pour participer aux États généraux d'Orléans (13 décembre 1560-31 janvier 1561) ${ }^{17}$.

Une curieuse mention du procès-verbal de la séance du 30 août 1560 signale une délégation précédente " aux États généraux de France qui se tinrent du vivant du feu roi Henri ". La formule est surprenante car il n'était pas dans le style d'Henri II de s'embarrasser d'une consultation des trois ordres de son royaume. Une allusion à des " lettres royaux " commandant son remboursement renvoie en fait à des lettres missives du 15 janvier 1557, produites dans la séance du 24 mars du même mois, relative à un voyage à la cour ${ }^{18}$. Comment interpréter cette formulation énigmatique autrement que par l'expression d'un manque de consultation des bonnes villes du royaume, signe de la décomposition rapide du modèle politique ${ }^{19}$ qui avait prévalu jusqu'à l'avènement de François Irr ? L'affaire est plus complexe car le conseil considère que cette députation a été accomplie au nom des États de Bretagne qui refusent pourtant de s'acquitter des frais ${ }^{20}$. La seule consultation connue renvoie à l'enquête ordonnée par Henri II à propos de l'implantation du parlement, mais il est impossible de la confondre avec des États Généraux. Il faut bien avouer que nous ne savons pas comment interpréter cette curieuse formulation.

Dans les délégations d'importance pour le port fluvio-maritime de la façade atlantique, incluons la participation à l'assemblée générale des marchands fréquentant la rivière de Loire se tenant régulièrement dans la ville d'Orléans. Le 5 avril 1557, sont envoyés le procureur des bourgeois, le contrôleur et François Salmon. Le choix des institutionnels souligne l'intérêt que porte la ville à l'entretien de la navigabilité du fleuve qui assure sa prospérité ${ }^{21}$.

Les députations s'étoffaient lorsqu'il s'agissait de négocier avec des commissaires royaux envoyés à Nantes, comme par exemple en avril 1558 lorsque la ville essaie vainement de faire réduire sa part de 24000 à 20000 livres dans la taxe de 120000 écus demandée à la Bretagne. Pour rencontrer les représentants royaux sont désignés 4 membres du clergé, 4 juges royaux, 4 délégués de la ville et 4 représentants des bourgeois ${ }^{22}$. Cette distinction entre

16. Arch. mun. de Nantes, BB $4, \mathrm{f}^{\circ} 76 \mathrm{r}^{\circ}$ et $74 \mathrm{v}^{\circ}$.

17. Arch. mun. de Nantes, BB 4, $\mathrm{f}^{\circ} 250 \mathrm{r}^{\circ}$.

18. Arch. mun. de Nantes, BB $4, \mathrm{f}^{\circ} 92 \mathrm{v}^{\circ}$.

19. ChEVALIER, Bernard, Les bonnes Villes de France du XIVe au XVIe siècle, Paris, Aubier, 1982, chap. 4 : La force politique. Le roi et les bonnes villes.

20. Arch. mun. de Nantes, BB $4, \mathrm{f}^{\circ} 242 \mathrm{v}^{\circ}$.

21. Arch. mun. de Nantes, BB $4, \mathrm{f}^{\circ} 94 \mathrm{v}^{\circ}$.

22. Arch. mun. de Nantes, BB 4, $\mathrm{f}^{\circ} 96 \mathrm{r}^{\circ}$. 
ville et bourgeois étonne dans un premier temps, mais se comprend immédiatement en observant la composition des deux groupes car elle correspond en fait à la dualité fondamentale de l'espace urbain nantais qui additionne une ville close très rétrécie (26 ha), rassemblant toutes les institutions, et un grand faubourg commercial en pleine extension ${ }^{23}$. Avec Julien Poullain, Yvon Rocaz, Pierre de La Presse et André Ruiz pour les bourgeois, il est certain que nous avons ici de bons représentants de l'élite des affaires de la Fosse, mêlant intimement grand commerce et spéculations financières ${ }^{24}$.

Les rapports du conseil des bourgeois avec le capitaine au château, le comte René de Sanzay, nommé en 1555, ont été très tendus dans la mesure où ce dernier a prétendu revitaliser sa fonction en restaurant des prérogatives tombées en désuétude. Le droit de visite des navires, l'organisation du guet et la nomination des portiers, l'attribution de la juridiction commerciale ont été des questions très controversées ${ }^{25}$. Dans la séance du 9 juillet 1558, s'organise une délégation pour trouver un accord sur le service du guet. Douze noms sont retenus afin de trouver six notables pour aller parlementer ${ }^{26}$.

L'émergence du calvinisme, la tension croissante entre les confessions rivales, les incertitudes liées à l'inflexion de la politique royale après la mort d'Henri II suscitent une grande inquiétude dans une ville profondément attachée au catholicisme, surprise de voir éclore en son sein une petite minorité huguenote, aussi bien dans les élites officières et marchandes que dans le monde artisanal et boutiquier ${ }^{27}$. Les notables se préoccupent de garantir la sécurité militaire de la ville, dans le loyalisme envers le pouvoir royal. Après le massacre de Wassy, le premier mars 1562, le déclenchement de la première guerre de religion les pousse à solliciter du gouverneur de Bretagne l'abandon de l'appel de la compagnie du seigneur de Goulaine en direction de Fougères, afin de garantir la défense de Nantes ${ }^{28}$. Il faut dire que la chaîne de succès huguenots dans les villes du Val de Loire avait de quoi inquiéter les Nantais ${ }^{29}$. Sont commis Jean Le Lou l'aîné, les deux de La Presse, Antoine de Mirande, Yvon Rocaz, François Salmon.

23. Thomas, Michèle, Topographie et habitants de Nantes au XV et au début du XVI siècle, DES, Nantes, 1965; DossEUL, Sandrine, Le quartier de la Fosse à Nantes aux XVI et XVII siècles, Maîtrise, SAuPIN, Guy (dir.), Nantes, 1996.

24. LAPEYRE, Henri, Une Famille de marchands, les Ruiz, Paris, SEVPEN, 1955.

25. VAILHEN, Jacques, op. cit., p. 370-392.

26. Arch. mun. de Nantes, BB $4, \mathrm{f}^{\circ} 121 \mathrm{v}^{\circ}$. "Le miseur, le controlle, François Salmon, Jean et Jean les Lou, Guillaume Catho, Pierre Cousin, François Amproux, Alain Marcraye, Jacques Gourdet, Yvon Rocaz, Antoine de Mirande. "

27. JoXe, René, Les Protestants dans le comté de Nantes (XVé-XVII siècles), Marseille, Laffite, 1982; SAuPIN, Guy, Nantes au temps de l'édit, 1560-1629, La Crèche, Geste Éditions, 1998.

28. Arch. mun. de Nantes, BB $4, \mathrm{f}^{\circ} 379 \mathrm{v}^{\circ}$.

29. Constant, Jean-Marie, Les Français pendant les guerres de Religion, Paris, Hachette, 2002 ; BouRQuIN, Laurent, Les nobles, la ville et le roi. L'autorité nobiliaire en Anjou pendant les guerres de religion (1560-1598), Paris, Belin, 2001. 


\section{Les délibérations en commission restreinte : le comité des douze}

Même si la sélection économique et sociale limite naturellement la participation au conseil des bourgeois à une étroite minorité d'habitants en grande partie issue du monde marchand, il est des occasions où la nécessité de délibérer en formation restreinte est fortement ressentie. La composition de ces commissions réduites est particulièrement intéressante à observer car il est logique de penser que les noms qui reviennent le plus souvent doivent désigner des personnes jouissant d'un prestige assuré ou disposant d'un réseau d'influence efficace. Le recours à un conseil de douze membres ${ }^{30}$ est régulier, même si les bourgeois prennent la précaution d'en sélectionner au moins deux fois plus, sans doute pour pallier les effets connus de l'absentéisme. Les sujets motivant ce recours à un conseil étroit sont divers. Toutefois, deux thèmes dominent de très loin la période analysée : la lutte contre Rennes pour l'obtention exclusive du parlement de Bretagne et la sûreté politique et militaire de la ville face à la montée des tensions confessionnelles.

Dans la séance du 27 janvier 1556, 8 marchands sont commis en sus des gens du roi et de la justice afin de délibérer sur les articles envoyés à la cour pour le règlement de la police des pauvres ${ }^{31}$. Les élections des deux administrateurs à l'aumônerie Saint-Clément relèvent aussi de telles commissions. Leur tâche n'est pas aisée car certains notables désignés refusent cette responsabilité, préférant s'acquitter d'un versement compensatoire $^{32}$.

Les oppositions avec le capitaine René de Sanzay ont fortement soutenu une telle pratique. Dans la séance du 2 février 1557, le conseil confie au miseur et à six autres marchands ${ }^{33}$ la mission de rédiger des "mémoires et instructions " à adresser au roi pour contrer la prétention du capitaine à visiter les navires entrant et sortant du port même si ces derniers ont déjà été contrôlés par les fermiers de la prévôté. Le représentant royal leur opposait l'obligation de contrôler le trafic d'armes ${ }^{34}$. Les contestations autour du service du guet, suite à l'exigence d'une présence de jour comme de nuit, avec levée d'amendes sur les défaillants " au prix de cinq deniers pour mois ", suscitent le même réflexe le 11 août 1558, même si 27 personnes sont mobilisées ${ }^{35}$.

30. Il est tentant de rapprocher ce comité restreint de douze membres de la composition initiale prévue par le duc Jean V en $1420: 12$ bourgeois dirigés par leur procureur venant s'associer aux principaux officiers ducaux (capitaine, connétable, sénéchal, prévôt, maître des monnaies) et six chanoines représentant l'évêque et les deux chapitres de la cathédrale Saint-Pierre et de la collégiale Notre-Dame.

31. Arch. mun. de Nantes, BB $4, \mathrm{f}^{\circ} 19 \mathrm{v}^{\circ}$.

32. CroIX, Alain, La Bretagne aux XVI et XVII siècles, la vie, la mort, la foi, Paris, Maloine, 1981, p. 579-592.

33. Arch. mun. de Nantes, BB 4, $\mathrm{f}^{\circ} 50 \mathrm{v}^{\circ}$. Le miseur, Mathurin Pelletier, Pierre de la Presse, Jean du Luc, François Amproux, François Salmon, Pierre Marcraye

34. VAILHEN, Jacques, op. cit., p. 381-387.

35. Arch. mun. de Nantes, BB 4, $\mathrm{f}^{\circ} 129 \mathrm{r}^{\circ}$. 
Après la victoire du groupe de pression nantais pour l'implantation exclusive du parlement provincial concrétisée par l'édit de juin 1557, le conseil des bourgeois veille à défendre cet avantage. Il faut d'abord décider du recouvrement de la finance utilisée. La commission mise en place le 5 décembre 1557 révèle une structure intéressante, presque conforme au schéma institutionnel originel, quoique valorisant les grands marchands de la Fosse ${ }^{36}$. Alors qu'il est " délibéré que deux de chacun des desdits trois états seront commis pour en communiquer ", l'ensemble formé comprend un vicaire représentant l'évêque, deux chanoines par chapitre, deux membres de la noblesse (distinction très inhabituelle), 6 bourgeois pour la ville et 3 pour la Fosse, l'alloué et le prévôt pour la justice et le procureur de l'Université. Le 5 juin 1558, il s'agit de contrer les réactions rennaises. Les capitaines et gens de justice, renforcés de douze des habitants, en sont chargés ${ }^{37}$.

La revanche de Rennes, qui a profité de la mort d'Henri II et des successions rapprochées pour renverser la situation sur l'attribution du parlement en mars 1561 grâce au soutien du gouverneur, le duc d'Etampes, provoque aussitôt la mobilisation nantaise. Le 27 avril, il est requis " qu'il soit député quelque nombre des habitants jusqu'au nombre de douze gens et personnes des suffisants de la ville qui pourront délibérer des affaires de la ville sans qu'il soit requis ordinairement faire congrégation et assemblée. Ayant mesdits sieurs sur ce délibéré ont ordonné que trente desdits habitants soient commis pour délibérer desdistes affaites, soit tant pour la suite du parlement contre les habitants de Rennes que pour la suite des récusations baillées contre monseigneur le gouverneur par Me Germain Bontemps leur procureur [...] et outre pour présenter requête à la cour afin d'avoir publication touchant un mandement et édit pour le fait de la religion que même pour envoyer au privé conseil du roi ${ }^{38}$ ". Dès le premier mai, un comité formé du sénéchal, de trois chanoines, du miseur et de douze habitants désignent trois d'entre eux pour faire le voyage de la cour, ce qui est soumis à validation à l'ensemble du Conseil dès la séance du 5 mai ${ }^{39}$.

La mise en défense de la ville afin de garantir sa sécurité conduit à un double réflexe selon la période et le type de menace. Le 10 septembre 1557, suite à la défaite catastrophique de Saint-Quentin devant les tercios espagnols, le capitaine, accompagné du connétable, s'est déplacé exceptionnellement afin de réactiver le système des gardes de la milice bourgeoise en plaçant cette mobilisation sous l'autorité d'un comité opérationnel. La formation de celui-ci montre comment le souci d'efficacité se double de la volonté de réunir l'ensemble des états représentatifs du conseil des bour-

36. Arch. mun. de Nantes, BB 4, $\mathrm{f}^{\circ} 78 \mathrm{r}^{\circ}$.

37. Arch. mun. de Nantes, BB 4, $\mathrm{f}^{\circ} 119 \mathrm{r}^{\circ}$. Neuf sont nommés de suite : Jean et Jean les Lou, Yvon Rocaz, Jacques Gourdet, Julien Poullain, Guillaume Poullain, Charles Chrestien, Alain Marcraye, François Amproux.

38. Arch. mun. de Nantes, BB 4, $\mathrm{f}^{\circ} 281$.

39. Arch. mun. de Nantes, BB $4, \mathrm{f}^{\circ} 282 \mathrm{v}^{\circ}, 283 \mathrm{r}^{\circ}$. 
geois de Nantes : chapitres, justice royale, bourgeois (marchands et auxiliaires de justice) :

"Monsieur le capitaine remontre que pour le fait des guerres, chose qui doit être secrète et non connue à plusieurs, l'on doit convenir de quelque nombre de gens tant de l'Église que de justice que habitants, afin d'ordonner et exécuter ce que par eux sera ordonné pour le fait desdites guerres, requiert qu'il en soit convenu de quelque nombre de gens de bien, et ayant lesdits habitants de ce conféré. A été délibéré que de chacune église de SaintPierre et de Notre-Dame, Messieurs de la justice et pour les habitants les officiers de la ville, Julien Poullain, les deux Loup, Yvon Rocaz, Jean et Pierre de La Presse, Louis et Guillaume les Poullain, Mathurin Pelletier, Mathurin Vivien, Simon Maillart, Charles Chrétien, François Salmon, Jean Picault, Pierre Cousin, Bertrand Duchesne, Aimé Loryot, Jacques Gourdet, Alain Marcrays, Léonard Le Vendangeur, Guillaume Nicollon, $\mathrm{M}^{\mathrm{e}}$ Guillaume Riallan, $\mathrm{M}^{\mathrm{e}}$ Robert Cauldron, Étienne Bouscher, M. de La Guillébaudière, MM. André de Boystual, Commaille, $\mathrm{M}^{\mathrm{e}}$ Jean Terrien, $\mathrm{M}^{\mathrm{e}}$ Claude Caillaud, Michel Jollen, Me Jean Delalande, Tullaye, $\mathbf{M}^{\mathrm{e}}$ de La Chauvière, Jean Guérin, François Forgil, Robert Pineau, Guillaume Frélon, Guillaume Pouponneau et dix ou douze d'eux ou le plus grand nombre que se pourra trouver, l'opinion desquels sera exécutée, et dès à présent lesdits habitants ont agréable ce que par eux sera fait et ordonné pour la garde et tution de la ville ${ }^{40}$."

Le recours au comité des douze devient systématique avec le déclenchement de la première guerre de religion. Dès le 31 mars 1562, les bourgeois, sous la présidence effective du capitaine, " ont convenu et accordé que douze des habitants nommés à la présente assemblée disposeront de toutes affaires en compagnie de M. le capitaine et gens de justice [...] à ce que les choses que l'ont fait aux assemblées ne soient divulguées et tenues secrètes $^{41}$ ".

L'ensemble de ces problèmes se conjugue pour provoquer la destitution du procureur des bourgeois Jean du Luc, marchand à la Fosse, le 22 août 1561. La multiplication des incidents entre la petite communauté réformée nantaise et la grande majorité des habitants se reflète dans une crise qui secoue le conseil des bourgeois puisque certains notables sont suspectés d'avoir rallié les hérétiques. Toutefois, l'affaire est gérée en douceur. Dès la séance du 10 août 1561, le procureur des bourgeois, suivi en cela par son substitut, demande à être remplacé. Le 22 août, le premier officier de ville est directement mis en accusation par les chanoines qui exigent sa destitution, en mettant en avant son absentéisme, sans aucune allusion à ses convictions religieuses. Dans la séance du 25 août, 17 habitants sont désignés pour statuer sur sa demande de retrait, aux côtés des représentants de l'Église et de la justice. Cette commission a certainement entériné la passation de pouvoir au profit du substitut Guillaume Catho puisque ce dernier agit comme procureur dans la séance suivante datée du premier octobre ${ }^{42}$.

40. Arch. mun. de Nantes, BB $4, \mathrm{f}^{\circ} 66 \mathrm{v}^{\circ}, 67 \mathrm{r}^{\circ}$.

41. Arch. mun. de Nantes, BB $4, \mathrm{f}^{\circ} 326 \mathrm{v}^{\circ}$.

42. Arch. mun. de Nantes, BB 4, $\mathrm{f}^{\circ} 287 \mathrm{r}^{\circ}, 289 \mathrm{r}^{\circ}, 291 \mathrm{r}^{\circ}$. "Pour la ville, Mathurin Vivien, François Salmon, Alain Marcraye, Étienne Bouscher, Colas Blanchard, Pierre Cousin, 
L'affaire s'éclaire un peu grâce aux protestations émises dans la séance du 11 novembre suivant où le nouveau procureur " remontre qu'aux États derniers tenus en cette ville un quidam se portant procureur des habitants de cette ville aurait accordé qu'il fut présenté requête au roi et à Mess. de son conseil privé afin d'avoir permission de faire un temple à la nouvelle loi que l'on dit et nomme la loi de Genève ". Scandalisés par une telle manœuvre, les bourgeois envoient un mémoire de protestation au roi. Cette hostilité à l'organisation d'un culte calviniste à Nantes s'exprime de nouveau dans la séance du 21 novembre, avec la même émotion dans la défense du catholicisme ${ }^{43}$.

La tonalité des lettres qu'Yvon Rocaz adresse à Simon Ruiz à Medina del Campo pendant la première guerre de religion en livre un éclairant témoignage. Après l'annonce de la mort du duc de Guise, vainqueur à Dreux mais blessé mortellement au siège d'Orléans, il se désole de cette perte immense : "Nous avons perdu le plus catholique et le plus vaillant qu'il y ait en France depuis mille ans. Que Notre Seigneur l'ait en sa gloire "; " Ce pauvre royaume est détruit, si Dieu n'y remédie pas à bref délai. " En 1568, dans la troisième guerre, il se fait plus tranchant. Parlant des huguenots, il déclare : "Beaucoup ont été défaits, mais ils naissent comme de la vermine ${ }^{44}$."

Tout en rappelant systématiquement leur loyalisme envers la monarchie, les bourgeois de Nantes ne manquent pas une occasion pour exprimer leur hostilité à toute tolérance, même limitée, du calvinisme dans le royaume ${ }^{45}$. Après l'enregistrement au parlement de Rennes de l'édit de coexistence de janvier 1562, l'émotion est forte dans la cité ligérienne. Dès le 6 mars, le suffragant de l'évêque

" remontre qu'il a été publié un édit à Rennes, à la publication duquel se seraient rendus opposants Monsieur de Nantes, Messieurs du chapitre et les habitants de Nantes, requérant lesdits habitants qu'ils aient à débattre qu'il soit fait article. A l'endroit a été par le procureur du roi dit et déclaré avoir charge desdits habitants de former opposition contre ledit édit et de fait lesdits habitants se sont opposés audit édit et pour poursuivre ladite opposition ont institué à leurs procureurs ceux qui par douze des habitants seront élus et choisis, et ont dès à présent nommé le miseur et 15 autres notables ".

Le procureur des bourgeois Guillaume Catho est député en cour à la fin de la séance ${ }^{46}$.

Guillaume Bretagne, Jean Gosset, Gravoil, Guillaume Ryallen, Blezeau, René Martin, Tullaye, Yvon Rocaz, Les Loup, Jean de La Presse et seront intimés à demain midi au château ".

43. Arch. mun. de Nantes, BB $4, \mathrm{f}^{\circ} 298 \mathrm{v}^{\circ}, 300 \mathrm{v}^{\circ}$.

44. LAPEYRE, Henri, op. cit., p. 404 . Lettres des 2 et 14 mars 1563 et du 3 décembre 1568.

45. Crouzet, Denis, La Sagesse et le malheur, Michel de l'Hospital, chancelier de France, Champvallon, 1998; Descimon, Robert, "Présentation », dans Michel de l'Hospital, Discours pour la majorité de Charles IX et trois autres discours, Paris, Imprimerie nationale, 1993; WANegfellen, Thierry (dir.), De Michel de l'Hospital à l'édit de Nantes. Politique et religion face aux Églises, Clermont-Ferrand, PU Blaise-Pascal, 2002.

46. Arch. mun. de Nantes, BB 4, $\mathrm{f}^{\circ} 324 \mathrm{r}^{\circ}$. En tête de liste viennent Yvon Rocaz, Jean et Pierre de La Presse, Pierre Cousin, Louis Poullain Antoine de Mirande. 


\section{Les finances : établissement des taxations et auditions des comptes}

Le contrôle des finances urbaines demeure parmi les meilleures garanties de la préservation d'une gestion collective au sein de l'oligarchie municipale. L'audition des comptes ordinaires du miseur s'inscrit comme une étape marquante de la gestion du conseil qui conserve sa structure tripartite fondamentale, à laquelle viennent s'adjoindre les représentants de la Chambre des comptes. Généralement, le rôle de vérification est dévolu à une dizaine d'habitants institués en Conseil. Ainsi le 5 août 1555, le procureur et quatrorze bourgeois s'ajoutent au représentant du capitaine, au sénéchal, au prévôt, mais aussi à deux maîtres aux comptes pour vérifier les comptes de Julien Poullain, miseur de 1546 à $1554{ }^{47}$.

\section{Le 7 janvier 1562, le procureur}

« remontre la présente assemblée avoir été ordonnée pour convenir et choisir des commissaires pour ouïr le compte du miseur, supplie qu'il en soit commis. Ayant lesdits habitants de ce conféré, ont commis pour Mess. des comptes, Mess. Motay et Morin. Outre Mess. le capitaine et gens de justice, sont commis ( 29 habitants) et dix d'eux, et assignation pour commencer audit compte demain à huit heures et sera continué de jour en autre ${ }^{48}$ ".

Le 8 janvier, huit bourgeois sont désignés pour examiner le minu en collaboration avec le prévôt et un chanoine de Saint-Pierre ${ }^{49}$. Reste d'ailleurs à justifier la somme de 1150 livres 9 sous 3 deniers sur le compte du précédent miseur Julien Poullain, dont il est demandé vérification à son fils Robert.

Les impositions extraordinaires exigées par le pouvoir royal sont autant d'occasion de réactivation de ce type de commission afin de négocier les meilleurs arrangements possibles avec le Trésor royal. En héritage du régime ducal, les villes closes de Bretagne étaient exemptes de fouages, équivalent breton de la taille, en échange de l'entretien de leurs fortifications ${ }^{50}$. Les ducs ne réclamèrent que de manière exceptionnelle des aides destinées au soutien des opérations de guerre (1434, 1477, 1487). Avec l'intégration progressive au royaume, les guerres d'Italie prolongées par le grand conflit contre les Habsbourg, ces exigences se firent plus lourdes et plus régulières ${ }^{51}$. De 1523 à 1562 , onze demandes royales ont correspondu

47. Arch. mun. de Nantes, BB 4, $\mathrm{f}^{\circ} 1 \mathrm{r}^{\circ}$. Derrière Mathurin Vivien, procureur des bourgeois, on trouve Pierre Bernard, Mathurin Pelletier, Julien Poullain, Geoffroy Drouet, Robin Pillays, François Salmon, Simon Maillart, Jean Courtoys, Jacques Gourdet, Pierre Marcray, Yvon Rocaz, Jean Duluc, Antoine de Mirande.

48. Arch. mun. de Nantes, BB 4, $\mathrm{f}^{\circ} 304 \mathrm{r}^{\circ}$.

49. Arch. mun. de Nantes, BB 4, $\mathrm{f}^{\circ} 305 \mathrm{r}^{\circ}$. Chrestien, Salmon, Blanchard, Bretagne, Panerot, Poullain, Juchaud et Guillaume Cousin, procureur des comptes.

50. La Nicolliere-Teijeiro, Stéphane de, Privilèges de la ville de Nantes, Nantes, 1883.

51. Le PAGe, Dominique, Finances et politique en Bretagne au début des temps modernes, 1491-1547, Paris, 1997. 
à un prélèvement d'au moins 88000 livres, avec un alourdissement très net à partir des années $1550^{52}$.

La levée des 23000 écus imposés à Nantes sur les 120000 écus attendus de la Bretagne nous permet de suivre les méthodes de négociation adoptées par le Conseil des bourgeois. Dans la séance du 6 avril 1558, après avoir entendu le président du parlement, agissant comme commissaire royal et accompagné des officiers de justice conduits par le sénéchal, expliquer que la somme que le roi fait lever sur les " aisés de tout le duché " est gagée par une rente sur le domaine royal amortissable en douze ans, le procureur des bourgeois propose de cotiser pour 12000 livres en unissant les efforts des bourgeois à ceux de l'Église, la noblesse étant taxée à part. Après avoir répondu ne pas pouvoir accepter moins de 15000 écus, "vu que la ville est une des plus meilleures villes dudit duché ", le président a quitté la salle, suivi du capitaine et du sénéchal. Les bourgeois ont alors décidé d'offrir 20000 livres tournois " pour tout le corps de la ville et faubourgs ", et " au cas que lesdits sieurs commissaires voudraient accepter l'offre, pour faire le contrat [...] ont choisi et député savoir pour l'église (4 chanoines), pour la justice (4 officiers) et pour les bourgeois, Julien Poullain, Yvon Rocaz, Pierre de La Presse, André Ruiz (la Fosse), pour la ville Robin Pillays, François Salmon, Étienne Bouscher, Charles Chrestien ${ }^{53}$ ". La séance du 22 avril, justifiée par l'arrivée du commis du traitant chargé du recouvrement, permet de comprendre que l'accord a finalement été passé à 24000 livres $^{54}$.

La traite foraine, sorte de taxe douanière frappant les marchandises sortant du royaume de France, établie par les rois à la fin du XIV e siècle, avait disparu après la transformation du duché en province. Pressé par les besoins d'argent, Henri II avait essayé de la rétablir en 1551, obligeant les États à négocier sa suppression par une offre de rachat dès la session de 1552, mais seulement acceptée en 1553 pour un montant de 132000 livres, pour lequel Nantes était cotisée à 19200 livres. Le conseil fut obligé de lever des taxes sur la circulation des marchandises afin d'amortir les emprunts contractés auprès des "plus aisés de la ville ". La délibération du 28 janvier 1554 arrêta la tarification. Selon les produits, le système de l'affermage coexista avec la levée directe par les professionnels ${ }^{55}$. Les comptes sont vérifiés selon la procédure traditionnelle, comme en février 1557 ou en mars $1558^{56}$.

52. VAILHEN, Jacques, op. cit., p. 157-170.

53. Arch. mun. de Nantes, BB 4, $\mathrm{f}^{\circ} 95 \mathrm{v}^{\circ}, 96 \mathrm{r}^{\circ}$.

54. Arch. mun. de Nantes, BB 4, $\mathrm{f}^{\circ} 100,101 \mathrm{r}^{\circ}$.

55. Arch. mun. de Nantes, BB 4, f $16 \mathrm{v}^{\circ}$. Séance du 15 décembre 1555. Voir les comptes dans CC 410.

56. Arch. mun. de Nantes, BB 4, $\mathrm{f}^{\circ} 86$. Les premiers et deux février 1557. Liste des marchands préposés à la levée des taxes. Sont nommés pour le contrôle : 14 bourgeois dont Charles Chrestien, François Salmon, Yvon Rocaz, Antoine de Mirande... Séance du 22 mars $1558\left(\mathrm{f}^{\circ} 157 \mathrm{v}^{\circ}\right)$ : le sénéchal, le procureur, le miseur et le controlle, Antoine de Mirande, Alain Marcraye, Pierre Marcraye, Pierre Cousin, Jacques Gourdet, Charles Chrestien. 
Lorsque le roi Henri II, en 1554, avait prétendu exiger des bourgeois une déclaration des fiefs nobles en leur possession afin de les soumettre aux francs fiefs, ceux-ci avait vivement protesté en rappelant l'ancienneté de leur privilège d'exemption (1331), ce qui avait obligé le roi à confirmer cet avantage, après enquête de la Chambre des comptes, dans ses lettres patentes données à Blois le 16 janvier 1556. Les lettres d'origine ne mentionnant que la ville, le conseil s'est préoccupé de proposer l'achat de l'exemption au bénéfice des faubourgs au prix de cent écus soleil. Dans la séance du 13 mai 1556, il fut convenu que la moitié de la somme devait être avancée par Françoise d'Astoudille, dame de Livernière, veuve de Jean de Compludo, important marchand d'origine castillane de la première moitié du XVI ${ }^{\mathrm{e}}$ siècle, pour une durée d'un mois, et l'autre moitié prise dans la miserie, sauf à recueillir la somme sur les bénéficiaires. Les députés en cour et les receveurs pour l'emprunt sont désignés dans la foulée ${ }^{57}$.

En réalité, pour obtenir satisfaction il a fallu monter jusqu'à 800 écus, accord ratifié par le conseil dans la séance du 21 juillet 1556, malgré l'opposition des grands marchands de la Fosse comme André Ruiz, Antoine de Mirande, Guillaume Poullain, Gratien d'Aragon, et Yvon Rocaz qui plaidaient pour un partage des charges, la moitié de la somme restant à la charge des bénéficiaires ${ }^{58}$. Les lettres patentes du 4 décembre 1556 ayant ordonné que soit repris le même type de taxation que pour le rachat de la traite foraine, l'exemption est bien généralisée. Le 11 septembre 1558, la dame de Livernière est obligée de produire des lettres du roi ordonnant son remboursement ${ }^{59}$. Le 22 janvier 1559, le procureur des bourgeois montre d'autres lettres du roi exigeant le versement complet des deniers promis pour le parlement et les francs-fiefs, ce qui conduit le conseil à désigner jusqu'à 18 bourgeois pour effectuer la vérification des comptes des nombreux receveurs ${ }^{60}$.

La question du financement de l'action de la ville auprès du conseil du roi pour l'obtention du parlement avait déjà fait l'objet d'une mise au point dans la séance du 25 juin 1557 où les bourgeois avaient accepté de garantir un emprunt de 13000 livres, portant intérêt à $10 \%$, auprès des plus aisés de Nantes ${ }^{61}$. Le lendemain, André Ruiz et le sire (Guillaume?) Poullain ont avancé chacun 1000 livres ${ }^{62}$.

La captation de la cour souveraine est une affaire si importante qu'elle conduit le conseil à s'élargir exceptionnellement en invitant le 22 octobre

57. Arch. mun. de Nantes, BB 4, $\mathrm{f}^{\circ} 27 \mathrm{r}^{\circ}$. Commis pour aller à la cour : deux sur quatre Guillaume Poullain, Jean Gillée, Jean Duluc, Robin Pillays. Sont commis pour recueillir l'argent 4 sur 7 désignés. François salmon, Pierre Bernard, Guillaume Poullain, $\mathrm{M}^{\mathrm{e}}$ Pierre Eveillard, Guillaume Bonfils, $\mathrm{M}^{\mathrm{e}}$ Guillaume morin, Georges Bernard.

58. Arch. mun. de Nantes, BB 4, $\mathrm{f}^{\circ} 33 \mathrm{r}^{\circ}$. Voir aussi Arch. mun. de Nantes, BB 3.

59. Arch. mun. de Nantes, BB $4, f^{\circ} 135 \mathrm{v}^{\circ}$.

60. Arch. mun. de Nantes, BB $4, \mathrm{f}^{\circ} 150 \mathrm{r}^{\circ}$.

61. Arch. mun. de Nantes, BB $4, \mathrm{f}^{\circ} 61 \mathrm{v}^{\circ}$.

62. Arch. mun. de Nantes, BB $4, \mathrm{f}^{\circ} 177 \mathrm{v}^{\circ}$. 
1557 les chefs des grandes familles nobles du comté ${ }^{63}$ à la discussion sur l'assiette des deniers qui doivent être levé. S'en suit, le 4 novembre 1557, la désignation d'une commission comprenant André Ruiz, Yvon Rocaz, Pierre de La Presse, Mathurin Pelletier, le miseur, le controlle, réunie chez l'évêque, avec le capitaine et les gens de justice. Cette procédure exceptionnelle est justifiée par la nécessité de faire contribuer l'ensemble du comté nantais ${ }^{64}$.

Le 5 décembre 1557, une assemblée particulièrement étoffée désigne une commission de tous les états sociaux du conseil, en distinguant encore représentations de la ville close et de la Fosse. L'assemblée du 9 décembre décide faire reposer la levée des 20000 livres sur le commerce des blés en levant une taxe de 50 sous tournois sur chaque tonneau " qui sera tiré hors de la comté de Nantes". Cette solution ne fait pas l'unanimité puisque dans le conseil du 15 décembre, le procureur des bourgeois, constatant que "les habitants ne se peuvent accorder, requiert qu'il soit commis et député quelque nombre de bons personnages pour voir la taxe qui en a été faite et y augmenter et diminuer à leur loyauté et conscience et de la forme de la cueillette desdits deniers ". Le 31 décembre 1557, Michel Loriot est alors député en cour pour obtenir le droit de lever 28000 livres " pour le parlement et leurs autres affaires ${ }^{65}$ ".

\section{Le noyau dur du Conseil des bourgeois}

\section{Les acteurs les plus influents}

En pointant les notables désignés dans les députations de la ville, dans les délibérations en comité restreint et dans les auditions des divers comptes, et en totalisant pour chacun le nombre de citations, nous devons approcher du cercle restreint de pouvoir dans lequel se mûrissaient les décisions importantes. Afin de respecter la marque de confiance supérieure attachée à une représentation de la ville vers les instances politiques extérieures, nous avons appliqué à cette mesure le coefficient 2 dans la constitution de notre tableau de sélection. En retenant comme seuil le chiffre de dix nominations coefficientées pour la période 1555-1562, ce dernier nous permet de dégager un nombre de 32 notables ayant bénéficié de la confiance de leurs pairs et marqué de leur engagement personnel l'action du Conseil des bourgeois. Ce noyau dur est loin d'être homogène dans la densité de présence; il renvoie plutôt à une hiérarchie à trois étages. Le seuil de 20 citations révèle une large base aux performances bien moyennes, représentant la moitié de l'effectif. L'autre moitié supérieure est elle-même répartie en deux sous-ensembles presque égaux de part et d'autre du seuil de 30 citations avec 7 exemples de 22 à 29 et 9 exemples de 36 à 54, ces derniers formant le cœur du Conseil.

63. Arch. mun. de Nantes, BB 4, $\mathrm{f}^{\circ} 70 \mathrm{r}^{\circ}$. $\mathrm{M}^{\mathrm{grs}}$ de Cargrones, de la Muce, de la Touche, de la Lymosinère, de Goullaynes, de la Fontaine

64. Arch. mun. de Nantes, BB 4, $\mathrm{f}^{\circ} 77 \mathrm{r}^{\circ}$

65. Arch. mun. de Nantes, BB $4, \mathrm{f}^{\circ} 78 \mathrm{r}^{\circ}, 80 \mathrm{r}^{\circ}, 82 \mathrm{v}^{\circ}, 84 \mathrm{r}^{\circ}$. 
Les marchands espagnols et l'administration de Nantes au milieu du XVI ${ }^{\mathrm{e}}$ siècle

Le noyau dirigeant du Conseil des bourgeois (1555-1562)

\begin{tabular}{|l|l|l|l|l|l|l|l|l|}
\hline Notables & Profession & Paroisse & Dép. & Com. & Fin. & Total & Mairie & Consuls \\
\hline Drouet Geoffroy M & Md. & St-Sat & 16 & 10 & 12 & 54 & M 65 & JC 66 \\
Rocaz Yvon & Md. Mer E. & St-Nic F & 11 & 15 & 16 & 53 & M $66-7$ & E 65 \\
Le Lou Jean aîné & Md. Mer E & St-Nic F & 16 & 9 & 6 & 47 & $\dagger$ & \\
Salmon François & Md. & St-Nic & 11 & 12 & 13 & 47 & E 65 & \\
Catho Guillaume P & Md. draps & Ste-Cr & 15 & 2 & 9 & 41 & & C66 J69 \\
Mirande Antoine & Md. Mer E & St-Nic F & 6 & 9 & 20 & 41 & E $65 \dagger$ & \\
Pillays Robin C & Md. & St-Sat & 13 & 7 & 7 & 40 & E 65 & \\
Duluc Jean P & Md. & St-Nic F & 12 & 5 & 8 & 37 & & \\
Chrestien Charles & Md. draps & St-Nic & 6 & 11 & 14 & 37 & & C 65 \\
Marcraye Alain & Md. draps & St-Sat & 3 & 8 & 15 & 29 & $\dagger$ & \\
Le Lou Jean jeune & Md. Mer E & St-Nic F & 5 & 10 & 5 & 25 & E 65 & \\
Cousin Pierre & Md. draps & St-Sat & 2 & 10 & 11 & 25 & E 66 & \\
Loriot Michel & Md. draps & St-Sat & 9 & 4 & 2 & 24 & E 65 & \\
La Presse Pierre de & Md. Mer E & St-Nic F & 6 & 6 & 5 & 23 & & \\
Vivien Mathurin P & Md. & Ste-Cr & 5 & 6 & 6 & 22 & & J 65 \\
Boucher Étienne & M. mercier & Ste-Cr & 2 & 10 & 8 & 22 & E 65 & \\
\hline
\end{tabular}

$\mathbf{P}$ : Procureur des bourgeois; $\mathbf{M}$ : miseur $; \mathbf{C}$ : Contrôleur $\mathbf{E}$ : Espagne; $\mathbf{F}$ : Fosse (port de mer); Dép. : Députations vers l'extérieur : la Cour, les États de Bretagne, le gouverneur, le capitaine; Com. : Comités restreints pour traiter des questions stratégiques; Fin : Commissions financières pour auditions de compte ou négociations de taxes; Total : Le chiffre est obtenu en appliquant le coefficient 2 à la colonne " députation "; $\mathbf{M} 65$ : Élu maire en 1565, E 65 : élu échevin, J 65 : élu juge Consul, C 65 : élu consul en 1565; † : décédé à la création de la première mairie

Bien sûr, ces données statistiques reflètent logiquement le poids des institutions dans la mesure où elles soulignent l'importance de deux des trois officiers du conseil. Le premier rang tenu par le marchand Geoffroy Drouet découle logiquement de sa fonction de miseur exercée de 1554 à 1564. Si la charge de procureur des bourgeois n'a pas été aussi efficace, alors qu'elle est dans son essence même la plus haute fonction de représentation de la communauté d'habitants, c'est tout simplement parce que la conjoncture du temps fait qu'elle a été assumée par trois personnes différentes pendant la période étudiée, d'abord Mathurin Vivien, marchand de draps de la paroisse Sainte-Croix (mars 1554-mai 1557), remplacé par le marchand à la Fosse Jean Duluc, destitué en octobre 1561, sans doute pour cause de sympathie pour la Réforme, et remplacé par son substitut Guillaume Catho, marchand drapier de la paroisse Sainte-Croix, en fonction jusqu'à l'installation de la nouvelle municipalité à la fin de 1564. Dans ces circonstances, il convient de signaler la très bonne position de Jean Duluc, et surtout de Guillaume Catho, ce dernier étant poussé en avant par la multiplication des problèmes lors de la naissance des guerres de Religion. Nul doute qu'en ramenant leur nombre de délégations au temps réel passé dans leur fonction dans la période étudiée, ils viendraient se placer logiquement en tête de notre sélection. La très bonne place de Robin Pillays, due essentiellement à ses nombreuses députations extérieures, renvoie 
également à ses fonctions administratives. Il a exercé la fonction de contrôleur de 1555 à 1564, alors que son père a rempli celle de miseur de 1536 à 1539, établissant sur deux générations une tradition de suivi personnel bien établie depuis la naissance du Conseil.

Au-delà de ces situations attendues où l'élection préalable à une charge administrative se traduit par un investissement majeur en temps de travail - ce qui montre au passage que ces offices urbains n'étaient pas d'aimables sinécures honorifiques, mais de bien lourdes responsabilités effectives l'approche la plus intéressante du point de vue de l'histoire sociale du politique renvoie surtout aux diverses prestations des notables qui ne sont en théorie que de simples citoyens agissant avec les autres dans une structure de pouvoir local sans règlement de fermeture. Le caractère oligarchique de la vie municipale au $\mathrm{XVI}^{\mathrm{e}}$ siècle cesse ici d'être une simple notion historique pour s'incarner dans des personnes dont la puissance économique et sociale était ainsi parfaitement reconnue et concrétisée.

Parmi les neuf notables du haut du tableau, six traduisent ainsi leur puissance financière et leur capital relationnel. Retenons d'abord l'excellent résultat consacrant Yvon Rocaz, grand marchand descendant d'une famille castillane immigrée à Nantes dès la première moitié du Xve siècle. Présent partout, il apparaît comme le chef d'orchestre quasi indispensable, capable de réunir l'ensemble des voix du Conseil des bourgeois. L'équilibre prévalant dans la ventilation de ses types de responsabilités le place au centre de l'exercice du pouvoir municipal nantais en ce milieu du Xvi ${ }^{\mathrm{e}}$ siècle, au cœur de la mobilisation du commerce dans son conflit avec le capitaine du château, à la tête de la dynamique qui a débouché sur l'obtention d'un corps de ville de type échevinal en 1559, mis en place en décembre 1564.

Viennent ensuite François Salmon, d'origine française, et Antoine de Mirande, d'origine castillane, tous deux marchands à la Fosse. Si le premier semble l'homologue de Rocaz tant leurs profils se ressemblent, le second se fait surtout remarquer par sa forte implication dans tout ce qui relève des questions financières. C'est un peu comme s'il était le garant des intérêts d'un certain nombre de grands marchands du commerce international, grands pourvoyeurs des emprunts relais régulièrement sollicités par le conseil. Nous retrouvons la même différence d'implication entre Jean Le Lou l'aîné, marchand à La Fosse, et Charles Chrestien, marchands de draps, tous deux de Saint-Nicolas. Le Lou se distingue par ses députations extérieures, surtout par une délégation aux États généraux d'Orléans tenus au tout début du règne de Charles IX, assemblée dans laquelle il fut envoyé non seulement par Nantes mais aussi par l'ensemble du tiers état de Bretagne, c'est-à-dire de la quarantaine de villes provinciales bénéficiaires du droit de députation. Chrestien n'a pas la même dimension politique puisqu'il émerge principalement par son fort investissement dans les comités restreints, principalement ceux traitant les questions financières. De ce fait, son type de participation évoque plus celui de Mirande. 
Derrière cet ensemble majeur, nettement dominateur dans notre comptage, se positionne un second groupe de personnalités dont le poids demeure néanmoins significatif. Dans l'ordre, relevons Alain Marcraye, marchand de draps de Saint-Saturnin, Jean Le Lou le jeune, frère cadet du précédent et lui aussi marchand à la Fosse, Pierre Cousin et Michel Loriot, marchands de draps de Saint-Saturnin, Pierre de La Presse, marchand à la Fosse, d'origine castillane, Etienne Boucher, marchand mercier de SainteCroix, et Mathurin Vivien, marchand, de la même paroisse. Seul le dernier bénéficie de l'effet administratif, pour lui-même ou pour son ascendance. Avant d'exercer la fonction de procureur des bourgeois de mars 1554 à mai 1557, Mathurin Vivien avait revêtu celle de contrôleur de 1540 à 1543, tandis qu'un de ses parents (père ou oncle?) avait déjà reçu la charge de miseur en 1525-1528.

Le trait caractéristique de cette seconde catégorie tient dans le fait que presque tous ces notables assoient leur présence active sur les commissions internes du Conseil, à l'exception singulière de Michel Loriot dont la présence à ce niveau est surtout due à ses nombreuses députations extérieures. Faut-il chercher dans le caractère ultra-catholique affirmé de cette famille un élément porteur dans une cité où la grande majorité des notables s'avère très hostiles à la Réforme et à toute forme de tolérance à l'égard des huguenots? Dans cette répartition entre députations externes et comités internes, certains présentent un profil plus équilibré que d'autres. Il en est ainsi de Jean Le Lou le jeune - ce qui traduit clairement la puissance sociale de cette famille en ce milieu de siècle - mais surtout de Pierre de La Presse qui semble reproduire, dans une échelle inférieure, la figure d'Yvon Rocaz. Les autres se sont distingués plus nettement dans les commissions intérieures, de manière assez équilibrée entre problèmes généraux et financiers, à l'exception d'Alain Marcraye qui doit sa place à une propension à être délégué aux contrôles des comptes.

Dans certaines consultations, il était habituel de distinguer les représentants de la ville close et du faubourg marchand de la Fosse. La répartition des principaux acteurs confirme assez bien la pertinence de cette disposition qui ne fait que refléter la distribution géographique du monde commercial nantais dans l'espace urbain. Les paroisses de Sainte-Croix (y compris la partie orientale de l'île de la Saulzaie) et de Saint-Saturnin perdaient progressivement les plus grands acteurs du commerce de mer au profit de Saint-Nicolas, tout en conservant les plus riches marchands détaillants, plus proches de leur clientèle nobiliaire et robine. Sur les 32 notables sélectionnés, 17 étaient domiciliés sur Saint-Nicolas, 7 sur SaintSaturnin, 3 sur Sainte-Croix et un sur Saint-Denis. En distinguant les trois niveaux observés, la prépondérance des grands commerçants de Saint Nicolas se révèle mieux, avec six exemples sur neuf au niveau supérieur, même si le niveau intermédiaire est au contraire très équilibré, la différence étant de nouveau creusée dans l'étage inférieur. 


\section{Acteurs majeurs du Conseil des bourgeois et réseau de parenté}

La liste des patronymes livre une première indication, somme toute superficielle. L'animation du Conseil n'est pas monopolisée par quelques familles qui y auraient pratiqué un entrisme systématique. Au contraire, il semble exister un consensus pour établir une réelle diversité mettant en correspondance la pratique cooptative de l'oligarchie avec l'esprit d'ouverture concrétisée juridiquement par l'absence de règlement de composition de l'assemblée générale des chefs de feu de la communauté d'habitants. Font malgré tout exception les trois Poullain, les deux Le Lou, les deux de La Presse, les deux Marcraye. Les trois frères Poullain n'apparaissent cependant que dans le niveau inférieur du tableau, révélant ainsi une implication personnelle directe limitée dans l'action municipale. Les deux frères de La Presse sont déjà plus actifs, sans atteindre l'impact des deux frères Le Lou, les seuls à agir dans les deux niveaux supérieurs de notre classement. Toutefois, on ne saurait en rester là. Tout historien qui s'est frotté à l'histoire sociale du politique sait bien qu'il est indispensable de reconstituer des réseaux de parenté dans lesquels les alliances matrimoniales viennent amplifier le capital social de chaque famille, même si cette distribution ne se fait pas à égalité. Cette reconstitution a aussi le mérite de faire apparaître d'autres familles qui ne sont pas directement partie prenante de l'action municipale, mais qui, par le biais de leurs parents, pouvaient peser sur les choix à effectuer.

Les trois frères Poullain sont les fils de Robin Poullain, marchand de Saint-Nicolas, et de Gratienne Arnollet, d'une famille d'origine castillane immigrée dès le milieu du $\mathrm{XV}^{\mathrm{e}}$ siècle. Leur père était suffisamment en vue pour être commis à l'audition des comptes de la miserie en 1517 et 1520 . Julien Poullain (1504-?), marié lui aussi dans la communauté hispanique avec Isabelle Houys (famille bien intégrée dans la paroisse Saint-Nicolas à la fin du Xv siècle), a exercé la charge de miseur de 1547 à 1554, signe de solidité financière d'une famille amenée à cautionner celui qui n'est pas seulement un trésorier mais surtout le premier prêteur d'argent au Conseil des bourgeois. Son civisme est indiscutable car au sortir de cette lourde charge, il accepte de tenir les comptes de l'Hôtel Dieu en 1554 en compagnie d'un autre marchand, Guillaume Bretagne, lui aussi distingué dans la base inférieure du classement. Sa position médiocre, étant données ses références antérieures, correspond peut-être à une fatigue physique de vieillissement qui se traduit par un retrait de la scène publique à partir de 1560. La reddition de son compte s'opère ainsi devant son fils Robert le 16 janvier $1560^{66}$.

Le plus impliqué paraît être Guillaume Poullain (1510-1591), marchand à la Fosse, marié à Jeanne d'Espinoze ${ }^{67}$, d'une famille ibérique bien implan-

66. Arch. mun. de Nantes, BB 4, $\mathrm{f}^{\circ} 256 \mathrm{r}^{\circ}$.

67. Jehanne, fille de Pierre d'Espinose et de Martine Billy ou de Billé, baptisée à SaintNicolas en 1522. 
tée à Nantes depuis la fin du Xv $\mathrm{Xv}^{\mathrm{e}}$ siècle avec deux branches développées à partir de Bernardin et de Pierre. Nous comptons 10 actes de baptême à Saint-Nicolas de 1546 à 1558. En 1567, il est inscrit en tête de la liste des principaux marchands consultés par le consulat du commerce de Nantes dans une enquête sur les affaires d'Espagne, nouveau tribunal corporatif dont il a été le premier consul à sa création en 1565. Capitalisant manifestement la notoriété de cette famille nombreuse ${ }^{68}$, comme l'indique le profil de son engagement laissant une bonne place aux députations extérieures, il semble pourtant en retrait des possibilités réelles du lignage et de son réseau de parenté, comme s'il se contentait de peser sur les choix de manière indirecte, par alliés interposés.

Louis Poullain (1498-?), époux de Marguerite d'Arande, renforce sa présence au Conseil dans les dernières années de la phase observée, surtout dans les questions financières. Pour être complet, mentionnons l'existence de deux autres frères, Jean (époux de Guyonne de Mirande) et Michel Poullain (époux de Françoise Le Mercier), qui ne font que de brèves apparitions dans les séances générales du Conseil. Ajoutons les trois sœurs : Guillemette, épouse de Robin Pillays l'aîné, miseur de 1536 à 1539; Marguerite, épouse de François Jallier, receveur ordinaire du domaine royal, et Gratienne, épouse de Gratien d'Aragon. Au total, sur huit enfants, cinq alliances dans les familles d'origine castillane contre trois seulement dans les familles françaises, les Le Mercier partageant d'ailleurs la même orientation. Il est évident que cette famille marchande aux ramifications très nombreuses a construit sa fortune en accordant toute l'attention nécessaire au premier horizon international du commerce nantais au XVI ${ }^{\mathrm{e}}$ siècle, avant de voir ses branches diversifier leurs parcours en faveur du négoce, de la finance ou des offices de justice ${ }^{69}$.

Nous pouvons considérer comme frères Pierre et Jean de La Presse puisque les actes de baptêmes de la paroisse Saint-Nicolas de juin 1518 et juillet 1521 nous renvoient à Jean de La Presse et à Jeanne de Mirande, les registres mentionnant les de La Presse depuis 1494. Cette famille était apparentée aux Ruiz et aux Mirande. Les liens demeuraient donc très étroits entre les branches castillanes et nantaises. La reconstitution des débuts de la famille Le Lou n'est pas aisée car les registres paroissiaux de SainteCroix et de Saint-Saturnin livrent plusieurs couples dont les liens sont délicats à établir, et ce depuis 1477. Ce qui semble le plus assuré, c'est que les deux frères sont les enfants du marchand Michel Le Lou et d'Amaurie Rouault (actes 1528, 1537), même si leurs actes de naissance demeurent pour le moment inconnus. Jehan Le Lou l'aîné s'est marié deux fois, d'abord avec Jeanne ou Marguerite Darande (citée en 1535, 1538), puis après son

68. SAUPIN, Guy, " Les Poullain, une dynastie municipale des XVI ${ }^{\mathrm{e}}$ et XVII ${ }^{\mathrm{e}}$ siècles ", Bulletin de la Société Archéologique et Historique de Nantes et de Loire-Atlantique, 1996, t. 131, p. 167-180.

69. SAUPIN, Guy, Nantes au XVII siècle. Vie politique et société urbaine, Rennes, PUR, 1996, p. 457 . 
veuvage (cité en 1553) avec Jeanne de Mirande (citée en 1560, mais veuve en 1564). Son frère cadet a épousé Jeanne Marboeuf (4 enfants de 1535 à 1543 sur Saint-Nicolas). Présenté comme veuf en août 1555, il est inhumé le 7 janvier 1566 à Saint-Saturnin ${ }^{70}$.

Geoffroy Drouet, sans relations parentales fortes à l'intérieur du noyau, tient donc essentiellement sa primauté de sa position institutionnelle comme miseur de la ville de Nantes. D'après son réseau familial, il apparaît d'ailleurs assez mal relié à l'élite du grand commerce nantais. Il en est de même des trois procureurs des bourgeois Mathurin Vivien, Jean Duluc et Guillaume Catho, avec des nuances pour le premier, beau-frère de Pierre Marcraye, parent d'Alain Marcraye, donc en lien avec une famille entrant dans l'orbite des Rocaz dans les années $1560^{71}$. Les spécialisations et les niveaux commerciaux n'expliquent pas tout. On peut comprendre que la situation de marchand de draps de la paroisse Sainte-Croix éloigne Guillaume Catho du grand commerce et que la diversité de position des deux Marcraye facilite au contraire un rapprochement (Pierre est marchand à la Fosse alors qu'Alain est marchand de draps à Saint-Saturnin), ce qui ne porte pas Jean Duluc, lui aussi marchand à la Fosse.

Le contrôleur Robin Pillays le jeune, pour le différencier de son père, s'inscrit dans une dynastie marchande établie sur les paroisses de SaintNicolas, mais surtout de Saint-Saturnin. Robin I, marié à Guillemette Poullain (7 actes à Saint-Nicolas, 1523-1535), s'inscrit donc dans l'orbite de cette grande famille marchande $\mathrm{du} \mathrm{XVI}^{\mathrm{e}}$ siècle déjà présentée. Robin II, marié à Jeanne Allaire, d'une famille marchande, (10 actes à Saint-Saturnin, 1541-1558), n'a pas recherché de liens de parrainage avec les familles d'origine espagnole, sans doute parce que ses activités renvoient plus au commerce de détail qu'au grand commerce de mer. Derrière les Poullain dominateurs viennent surtout des familles marchandes de seconde catégorie comme les Brenezay ou les Bourdin. Ces dernières alliances sont cependant loin d'être négligeables car elles ouvrent sur un réseau qui a fourni ensuite deux maires de Nantes ${ }^{72}$, avec Jean Fruneau (1586-1587) et Guillaume Du Bot (1595-1596).

Tout change par contre lorsque nous passons à l'examen des réseaux de parenté des personnalités les plus actives du Conseil, hors fonction officielle. L'exemple le plus éclatant en est fourni par Yvon Rocaz, dont le choix des alliances pour ses enfants correspond à la période analysée. Les liens sont très forts avec les Ruiz grâce aux mariages croisés de Julien Rocaz et de Marie Ruiz en 1564 et de Jeanne Rocaz et de Julien Ruiz en 1565, deux

70. Arch. dép. de Loire-Atlantique, 25 J, Fonds Freslon, Nantes tome XI. Cette base de données essentielle est dorénavant informatisée.

71. Mariage de Jean Rocaz, sieur de l'Abbaye, fils d'Yvon, avec Jeanne Marquerays. Baptêmes de leurs enfants Marie et Jeanne le 12 décembre 1564 à Saint-Nicolas.

72. SAupin, Guy, La Vie municipale à Nantes sous l'Ancien Régime, 1565-1789, Thèse de $3^{\mathrm{e}}$ cycle, dact., Université de Nantes, 1981, 2 vol., p. 310-312, 332-334. 
des enfants du grand marchand et financier André I Ruiz ${ }^{73}$, lui-même marié à Isabelle de Sancto-Domingo depuis au moins 1540 .

Les autres alliances renvoient vers les Le Lou, avec le couple Françoise Rocaz-Michel Le Lou (vers 1560), marchand, receveur des fouages de l'évêché de Tréguier avant de devenir maître aux Comptes en 1572, fils de Jean Le Lou le jeune et neveu de Jean Le Lou l'aîné, mais aussi vers les Mirande, suite au remariage en secondes noces vers 1571 de Julien Rocaz avec Catherine de Mirande, fille de Diego de Mirande et de Marie de Mirande. Les liens d'affaires avec les Ruiz expliquent l'alliance vers 1568 de Marguerite Rocaz et Nicolas Fyot, receveur des fouages de l'évêché de Tréguier ${ }^{74}$. Rappelons aussi les Marcrays déjà cités.

Nicolas Fyot a été formé au commerce en travaillant comme facteur à Bilbao (1560-1561) pour le compte de Simon Ruiz, le grand brasseur d'affaires de Medina del Campo. Après être revenu à Nantes pour trafiquer à son compte, il a refusé en 1568 d'entrer dans la société formée par son ancien maître avec Yvon Rocaz et Michel Le Lou, en invoquant le fait que Simon Ruiz ne s'intéressait qu'aux importations de France vers la Castille, alors que lui-même était très actif dans les retours en fer en provenance de Biscaye. Cela n'empêcha pas tous ces entrepreneurs de rester fort proches comme en témoigne la prise en ferme du bail de la prévôté de Nantes en 1579 partagé entre André Ruiz, Julien Rocaz et Nicolas Fyot, ce dernier ayant déjà bifurqué vers les finances en acquérant l'office royal de receveur de fouages ${ }^{75}$.

Il est très délicat de reconstituer avec exactitude le réseau de parenté des Mirande ${ }^{76}$, tant les indications des registres paroissiaux sont fragmentaires et les homonymies fréquentes. L'implantation nantaise est ancienne, ce dont témoigne la présence de Martin I de Mirande cité comme marchand d'Espagne en 1477 dans le registre de Saint-Nicolas, mais aussi d'Alonso de Mirande, époux de Guyonne Le Gouz (4 actes à Saint-Nicolas, 1494-1500), parrain pour un fils de Martin de Mirande (le même ou son fils?). Leur est lié Gracian de Mirande, cité en 1500. À la génération suivante, nous trouvons Hiérosme de Mirande, l'époux de Guillemette Baugé, mais aussi Julien, " chanoine de Nantes ", et François, prieur d'Indre. Les premiers sont les parents de Guyonne, née en 1522, épouse de Jean Poullain (8 actes à SaintNicolas 1540-1547), et de Jacquette, née en 1526, épouse de Jacques de Villadiego. Deux autres couples sont plus délicats à positionner. Nous sommes tentés de considérer Jacques de Mirande, dit aussi Diego, comme

73. LAPEYRE, Henri, op. cit., p. 47-59. André Ruiz, dont la présence nantaise est attestée depuis 1537, aurait commencé comme employé chez Francisco de Sancto-Domingo, marchand castillan installé à Nantes dès 1522, dont il aurait épousé la fille, selon une hypothèse avancée par H. Lapeyre. Il a obtenu ses lettres de naturalité en mars 1546.

74. Ibidem, p. 112. Lettre de Yvon Rocaz à Simon Ruiz, 13 mars 1568.

75. Ibid., p. 50 .

76. Arch. dép. de Loire-Atlantique, 25 J, Fonds Freslon, Nantes. Toutes les observations viennent de la consultation de ces fiches. 
leur frère, tandis que son épouse Marie de Mirande pourrait être la sœur d'Antoine de Mirande, frère ou cousin de Hiérosme, car le sentiment familial est explicite entre toutes ces personnes. Ainsi, notre acteur majeur du Conseil, marié à Marie du Val, d'une famille marchande nantaise (11 actes à Saint-Nicolas, 1537-1554), décédé en mai 1565, a construit un réseau de relations croisant familles françaises et castillanes, avec des liens privilégiés avec les Du Val et les Poullain d'une part et les d'Espinose d'autre part.

Par Catherine, fille de Jacques et Marie, seconde épouse de Julien Rocaz vers 1571, les Mirande se connectent au réseau de parenté Rocaz-Ruiz déjà évoqué ci-dessus. Nous hésitons sur la filiation de Jeanne de Mirande, seconde épouse de Jean Le Lou l'aîné à partir de 1560. Enfin, comment rattacher une autre Jeanne de Mirande, mariée à Pierre Cheminart, citée en 1564, en parenté par son époux avec les de Compludo? Notons que les enfants d'Antoine de Mirande, mariés après le décès de leur père, ont trouvé prioritairement leurs conjoints dans ce milieu marchand hispanonantais : Antoine avec Gatianne d'Aragon en 1578, Ferrando avec Françoise d'Aragon vers 1583. Toutefois, Louise a été mariée en 1583 à Antoine de Brenezay, avocat du roi au présidial de Nantes, futur sénéchal ligueur de Nantes en rébellion contre Henri IV.

\section{Le réseau de sociabilité d'Antoine de Mirande}

\begin{tabular}{|l|l|}
\hline Parenté par alliance & Réseau des parrains et marraines \\
\hline Du Val & Marie Poullain * Husquin du Val \\
\hline De La Presse & Jeanne Poullain * Jacques du Val \\
\hline Complude par les Le Gouz & Jehan, Gracianne et Marguerite du Val \\
\hline Baugé & Gracianne Arnollet, veuve de Robin Poullain (2) \\
\hline Poullain & Guillaume Poullain * Jeanne d'Espinose \\
\hline Villadiego & Julien, Michel, Thomasse Poullain \\
\hline & Pierre d'Espinose l'aîné (3) \\
\hline & Marie (2), Jeanne, Marguerite, filles de Bernardin d'Espinose \\
\hline & René et Pierre le jeune, fils de Bernardin d'Espinose \\
\hline & Ferrando d'Espinose \\
\hline & Jacques d'Espinose, fils de Pierre l'ainé (3) \\
\hline & Isabelle d'Espinose, fille de Pierre l'aîné \\
\hline & Isabelle de Santo-Domingo * André Ruiz \\
\hline & Gratien d'Aragon * Gracianne Poullain \\
\hline & Françoise Vivien * Pierre Marcrays \\
\hline
\end{tabular}

François Salmon, autre acteur majeur très engagé dans la conduite du Conseil, n'apparaît qu'épisodiquement en tant que de notable sollicité par d'autres dans le système relationnel de l'oligarchie municipale, sans doute parce qu'il est le dernier représentant, demeuré célibataire, d'une famille marchande présente à Saint-Nicolas au début du XvI ${ }^{\mathrm{e}}$ siècle. Peut-on voir dans cette absence de responsabilité familiale une bonne disponibilité investie au service de la communauté? 


\section{Les réseaux d'affaires : \\ le marché castillan et les fermes d'impôt de Bretagne}

Il est assez classique de constater que les réseaux de parenté recoupent en large partie les réseaux d'affaires, sans se superposer rigoureusement. Nos exemples en fournissent une nouvelle illustration. Grâce à Henri Lapeyre, les liens commerciaux unissant plusieurs de nos familles autour des Ruiz sont connus depuis longtemps. Jusque vers 1630, la péninsule ibérique est restée le premier horizon international du port de Nantes ${ }^{77}$, grâce aux exportations de toiles de chanvre et de lin de l'Ouest français et aux importations de laine et de produits métallurgiques du Pays basque. La relance des guerres entre Henri II, Charles Quint et Philippe II dans les années 1550 ont incité les Ruiz et les de La Presse à former une société commerciale unissant son frère Vitores Ruiz, de Medina del Campo, ville des grandes foires de Castille, et son cousin Francisco de La Presa, de Burgos, métropole de la laine et des assurances, à André Ruys, le plus important des marchands d'origine castillane installés à Nantes, et à Jean de la Presse ${ }^{78}$.

Par ailleurs, Simon Ruiz, frère d'André et de Vitores, puissant marchand et financier de Medina del Campo, travaillait en collaboration avec Yvon Rocaz dès 1545 en ce qui concerne l'expédition des toiles de chanvre et de lin de l'Ouest de la France et la réception des ballots de laine de Castille et du fer du Pays Basque en échange. La guerre a perturbé ce trafic, mais tous les moments de répit ont été mis à profit, comme en 1554 où Yvon Rocaz relance les expéditions avec le soutien de Jean Le Lou le jeune ${ }^{79}$. La trêve de Vaucelles de juin 1566 a incité Simon Ruiz à créer une véritable compagnie de commerce avec ses deux partenaires nantais ${ }^{80}$, chacun apportant le tiers du capital, association renouvelée tous les trois ans et simplement interrompue par la mort d'Yvon Rocaz en 1569. Le décès de Jean Le Lou n'avait pas eu le même effet en 1566, son fils Michel ayant immédiatement pris le relais. Il faut dire que Julien Rocaz, son fils aîné, s'intéressait plus aux spéculations proprement financières, comme le montrent ses acquisitions de l'office de receveur des fouages de l'évêché de Cornouaille en 1571, suivi de celui de trésorier et receveur général des rentes royales en Bretagne en 1582, à la suite de Vital de Contour dont il épousa la fille en novembre 1577.

77. PRIOTTI, Jean-Philippe, « Nantes et le commerce atlantique : les relations avec Bilbao au Xvi siècle ", Annales de Bretagne et des Pays de l'Ouest, 1993, t. 100, n 3 . CASADo Alonso Hilario, "Le commerce des " marchandises de Bretagne " avec l'Espagne au XvI siècle ", Annales de Bretagne et des Pays de l'Ouest, 2000, t. 107, n², p. 29-50.

78. LAPEYRE, Henri, op. cit., p. 48. La mort des associés Vitores en 1566, Jean de la Presse en 1567 et Francisco de la Presa en 1577 n'empêcha pas la compagnie de subsister, les affaires étant poursuivies par les héritiers.

79. Arch. mun. de Nantes, Nantes, HH 190, $\mathrm{f}^{\circ} 31$ sqq.

80. TANGUY, Jean, « L'exportation des toiles de la France de l'Ouest en Espagne dans la seconde moitié du XVI ${ }^{\mathrm{e}}$ siècle ", dans SANCHEZ, Jean-Pierre (dir.), Dans le sillage de Colomb. L'Europe du Ponant et la découverte du Nouveau Monde (1450-1650), Rennes, PUR, 1995, p. $193-200$. 
À partir de 1569, Julien Ruiz, gendre de Rocaz depuis 1565, formé aux affaires à Medina del Campo sous l'autorité de son oncle Simon, désireux de s'émanciper à Nantes de la tutelle paternelle, obtint de s'occuper directement des affaires de son oncle. Dans cet ensemble d'opérations commerciales et financières, tout gravite autour de Simon Ruiz ${ }^{81}$ qui travaille aussi en 1554 avec Vitores Ruiz et Francisco de la Presa, les partenaires castillans de son frère André.

La période analysée s'inscrit dans la première phase des activités financières des partenaires, lorsque ceux-ci se limitaient à l'affermage de collectes de taxes locales comme le denier pour livre en 1548 (bail de 4000 livres) ou le bail de la prévôté en 1565. La saisie de fermes d'envergure supérieure comme les impôts et billots de Bretagne, dans laquelle entraient les revenus des péages royaux provinciaux, ne date que de 1576. Cette montée en puissance a certainement été soutenue par l'expérience acquise et les profits recueillis dans le courtage au service de la banque lyonnaise, et tout spécialement des Bonvisi.

En dehors de ces associations formelles, les relations entre tous ces grands marchands orientés vers l'horizon castillan étaient favorisées par la mise en pratique des accords de " contractation " entre les ports de Nantes et Bilbao ${ }^{82}$. En préfiguration à une association structurée dont il n'existe aucun témoignage archivistique avant 1601, un fonctionnement coutumier placé sous l'autorité du consul d'Espagne à Nantes rapprochait tous les marchands désireux d'expédier des marchandises vers les ports de la côte cantabrique. Malheureusement, il ne reste plus que quelques exemples isolés de cette régulation, tant en 1567 par le biais d'un procès intenté par un marchand de Rennes contre "Astoudille, pour lors consul de la Nation d'Hespaigne ", qu'en 1584 grâce à une lettre d'André II Ruiz adressé à son oncle Simon. Les chargements collectifs rassemblaient ainsi tous nos principaux acteurs. En juillet 1559, le conflit entre ces marchands et le capitaine du château, le comte René de Sanzay, a débouché sur un procès, crise fort utile pour notre documentation ${ }^{83}$.

Ce court essai d'histoire sociale du politique appliqué à une grande ville commerciale française du XVI ${ }^{\mathrm{e}}$ siècle dont la montée en puissance était encore à venir et dont le principal horizon international était encore le

81. LAPEYRE, Henri, op. cit., p. 65. En juillet 1565, Simon Ruiz, André Ruiz et Yvon Rocaz se sont retrouvés à Bayonne pour dresser le bilan de leurs deux compagnies de commerce. Le chiffre d'affaires calculé est considérable : 162134843 maravédis " pour 9404 pièces de toute sortes, fardeaux de lingeries et paniers de cardes venus de Bretagne ", auxquels il faut ajouter 17762220 maravédis de marchandises de Rouen.

82 JeuLin, Paul, "Aperçus sur la Contractaction de Nantes, 1530 environ-1733 ", Annales de Bretagne, 1932-1933, tome XL, p. 284-331; LAPEYRE, Henri, op. cit., p. 228.

83 Arch. mun. de Nantes, EE 244, 12-15. Dans le conflit autour du navire de Pierre Colin apparaissent quelques acteurs qui nous intéressent comme " Anthoine de Myrande, 20 fardeaulx pappier, huit pannyer des quardes ou encore Garcia d'Aragon, 3 fardeaulx de mèche pour faire chandelle et 17 pannyer des quardes ". 
royaume de Castille permet de dégager trois grandes observations. Avant la création de la municipalité sous forme d'échevinage, le pouvoir urbain est passé entre les mains de l'oligarchie marchande qui a pris l'habitude de le gérer collectivement. Cette forme d'action a influencé ensuite le mode de gestion du corps de ville dont elle a voulu la création et permis l'installation pour mieux préserver son autonomie d'action dans le commerce international. L'intégration des grandes familles castillanes dans cette oligarchie est manifeste puisque plusieurs individualités les représentant parfaitement sont bien positionnés dans le cercle étroit le plus influent pour la conduite des affaires urbaines. L'élection ultérieure de maires provenant du même milieu est venue confirmer la solidité de cette implantation. L'analyse des réseaux d'influence illustre de façon exemplaire la solidité de cette élite mixte, étrangère et indigène, quant aux origines géographiques, ainsi que l'élargissement du milieu dirigeant apte à peser sur les choix politiques majeurs par relations interposées. En sus du fait de sa puissance financière, André I Ruiz n'avait pas besoin de siéger au Conseil pour y être quand même très présent.

\section{RESUME}

Organisé en 1420, Le Conseil des bourgeois s'est occupé des affaires municipales sous la tutelle ducale, puis royale jusqu'en 1564. Au Xvie siècle, il fonctionnait sous la forme d'une assemblée générale, dotée d'un petit exécutif formé d'un procureur des bourgeois, d'un miseur et d'un contrôleur. Le véritable pouvoir était détenu par les grandes familles qui siégeaient régulièrement et qui cumulaient les missions essentielles : députations extérieures, contrôle financier, comité des douze... Dans cette oligarchie marchande, les familles d'origine espagnole (Rocaz, Mirande, La Presse) en voie d'intégration dans la société nantaise ont tenu une place minoritaire, mais essentielle, basée sur leur bonne intégration dans les réseaux marchands.

\section{ABSTRACT}

Constituted in 1420, the freemen's Council directed the municipal policy under the ducal and royal control up to 1564 . In the $16^{\text {th }}$ Century, it squared with a general and oligarchic meeting, conducted by a little executive made of a freemen's procurator, a treasurer and a controller. The real power was in the hands of rich families who regularly sat and held a plurality of important missions : outside deputations, financial control, committee of twelve... In this merchant oligarchy, the spanish families in way of integration into the society of Nantes occupated a minority but important position, based on a very good integration in the merchant networks. 